\title{
Does Multilevel Lumbar Stenosis Lead to Poorer Outcomes?
}

\author{
A Subanalysis of the Spine Patient Outcomes Research Trial \\ (SPORT) Lumbar Stenosis Study
}

\author{
Daniel K. Park, MD, ${ }^{*}$ Howard S. An, MD, ${ }^{*}$ Jon D. Lurie, MD, MS, † Wenyan Zhao, MS, $\neq$ \\ Anna Tosteson, ScD, †\$ Tor D. Tosteson, ScD, $\mathbb{S}$ Harry Herkowitz, MD, 9 Thomas Errico, MD,\| \\ and James N. Weinstein, DO, MSt
}

Study Design. A subanalysis study.

Objective. The purpose of this study was to determine the impact of multilevel lumbar stenosis with or without degenerative spondylolisthesis compared to single level disease on patients' baseline symptoms and clinical outcomes over time.

Summary of Background Data. Previous studies have demonstrated better clinical outcomes with surgery than nonoperative treatment in patients with spinal stenosis with or without degenerative spondylolisthesis. However, the impact of multilevel stenosis has not been studied in these patients.

Methods. The results from a multicenter randomized and observational study, the Spine Patient Outcomes Research Trial (SPORT) comparing surgical versus nonoperative treatment for spinal stenosis with or without spondylolisthesis, were analyzed. The primary outcomes measures were the Bodily Pain and Physical Function scales of the Medical Outcomes Study 36-item Short-Form General Health Survey (SF-36) and the modified Oswestry Disability Index at 6 weeks, 3 months, 6 months, 1 year, and 2 years. Secondary outcome measures included the stenosis bothersomeness index, leg pain bothersomeness, low back pain bothersomeness, and patient satisfaction.

Results. In this subanalysis of SPORT data, multilevel spinal stenosis did not demonstrate worse baseline symptoms or worse treatment outcomes in isolated spinal stenosis; however, if concomitant degenerative spondylolisthesis existed, patients with only single level stenosis tended to improve more than those with multilevel stenosis, particularly after surgery.

From the *Department of Orthopaedic Surgery, Rush University Medical Center, Chicago, IL; Departments of †Medicine, $\ddagger$ Orthopaedics, and $\$$ Community and Family Medicine, Dartmouth Medical School, Lebanon, NH; IIWilliam Beaumont Hospital, Royal Oak, MI; and |New York University- Hospital for Joint Diseases, NY, NY.

Acknowledgment date: November 12, 2009. Revision date: May 4, 2009. Acceptance date: May 26, 2009.

The manuscript submitted does not contain information about medical device(s)/drug(s).

Federal funds were received in support of this work. No benefits in any form have been or will be received from a commercial party related directly or indirectly to the subject of this manuscript.

The National Institute of Arthritis and Musculoskeletal and Skin Diseases (U01-AR45444) and the Office of Research on Women's Health, the National Institutes of Health, and the National Institute of Occupational Safety and Health, the Centers for Disease Control and Prevention. The Multidisciplinary Clinical Research Center in Musculoskeletal Diseases is funded by NIAMS (P60-AR048094).

Address correspondence and reprint requests to James N. Weinstein, DO, MS, The Dartmouth Institute for Health Policy and Clinical Practice; Department of Orthopaedics, One Medical Center Dr., Lebanon, NH; E-mail: sport@dartmouth.edu
Conclusion. Patients with spinal stenosis without associated degenerative spondylolisthesis or scoliosis can be managed nonoperatively irrespective of the number of levels involved. If surgery is performed, the number of levels treated does not predict outcome. In contrast, patients with concomitant degenerative spondylolisthesis and single level stenosis do better surgically than those with additional levels of stenosis. This study emphasizes the importance of shared decision-making between the physician and patient when considering treatment for spinal stenosis.

Key words: spinal stenosis, degenerative spondylolisthesis, lumbar decompression, clinical outcomes. Spine 2010;35:439-446

Lumbar spinal stenosis (SpS) is defined as narrowing or stricture of the spinal canal. In some patients, this compression becomes symptomatic; the classic presentation is that of bilateral neurogenic claudication defined as intermittent pain radiating to the buttocks/thigh and/or leg that is worse with prolonged standing, walking, or lumbar extension. However, many individuals remain asymptomatic, and radiographic findings do not necessarily correlate with clinical symptoms. ${ }^{1,2}$ Lumbar SpS occurs with normal vertebral alignment; however, some patients also suffer from concomitant degenerative spondylolisthesis (DS). DS is defined as the forward slipping of one lumbar vertebra on another with an intact neural arch. Most DS affects the L4-L5 level. ${ }^{3,4}$ It commonly occurs in patients over the age of 50 and affects females $6: 1 .^{5}$ DS is generally asymptomatic, but can be associated with symptomatic $\mathrm{SpS}$ and radiculopathy. ${ }^{4}$

When patients suffer from symptomatic SpS, irrespective of concomitant DS, treatment options include either surgical or nonoperative measures. Outcome studies comparing surgical treatment to nonoperative measures have been performed. The Maine Lumbar Spine Study demonstrated superior surgical outcomes at 1 and 4 years. At 8 to 10 year follow-up, low back pain relief, predominant symptom improvement, and satisfaction with current state were similar between the 2 groups. Leg pain relief and greater back-related functional status, however, favored surgical intervention. ${ }^{6-8}$ Amundsen et al also reported a prospective study where after 4 years, excellent or fair results were found in $50 \%$ of the nonoperative patients, whereas excellent or fair results were found in $80 \%$ of surgical patients. ${ }^{9}$ More importantly, 
predictors of who would benefit from surgery or nonoperative measures have been elusive.

One potential predictive factor may be the number of stenotic levels. To date, no study has thoroughly compared the results of nonoperative versus surgical options in patients with isolated 1 level $\mathrm{SpS}$ compared to multilevel SpS. Furthermore, no study has compared systematically if surgery and nonoperative outcomes are superior for 1 level SpS with DS compared to multiple levels.

Recently, a multicenter randomized and observational trial, the Spine Patient Outcomes Research Trial (SPORT), compared surgical versus nonoperative treatment for SpS without spondylolisthesis or scoliosis. ${ }^{10}$ Although there was a high level of nonadherence in the randomized groups, this study demonstrated significantly more improvement with operative treatment in all primary outcomes than nonoperative treatment when an as-treated analysis was performed. This benefit appeared at 3 months and remained significant up to 2 years. ${ }^{10}$ In a separate study arm, the SPORT trial also examined patients with $\mathrm{SpS}$ and associated DS. ${ }^{11}$ Similar to the SpS group, a high level of crossover was found. In an astreated analysis, surgical treatment substantially demonstrated greater improvement in pain and function during the 2 -year collecting period. ${ }^{11}$

In the current study, subanalyses of SPORT for isolated SpS with normal vertebral alignment and SpS with associated DS were performed to determine the impact of multilevel SpS compared to single level disease on patients' baseline symptoms and clinical outcomes over time. These analyses represent the first clinical study comparing the presentation and treatment outcomes of 1,2 , and multilevel lumbar SpS in patients with and without associated DS.

\section{- Materials and Methods}

\section{Patient Population}

Spinal Stenosis Group. At 13 spine clinics in the United States, 289 patients were enrolled in a randomized cohort, and 365 patients were enrolled in the observational cohort out of a total of 1091 patients eligible for enrollment. Each patient demonstrated a history of at least 12 weeks of symptoms and radiographically had confirmed $\mathrm{SpS}$ without DS or associated scoliosis. Treatment was either decompressive surgery or usual nonoperative care defined by each institution. In total, 400 patients received surgery, and 254 remained in nonoperative management. Of the 654 patients, 634 had at least 1 follow-up through 2 years. Fifteen patients were excluded as they did not have data on the number of moderate/severe levels, leaving 619 patients in the current analysis.

Degenerative Spondylolisthesis Group. Patients who also had at least 12 weeks of symptoms and radiographic confirmation of SpS and with the addition of an associated DS were offered enrollment into the separate degenerative spondylolisthesis arm of SPORT. DS was diagnosed on standing lateral radiographs. Patients with isthmic spondylolisthesis were excluded. Patients with only 1 level of spondylolisthesis were included. Treatment was either decompressive surgery with or without fusion or usual nonoperative care. Overall, 607 of 892 eligible patients were enrolled; 304 patients were placed in the randomized cohort and 303 patients in the observational cohort.

\section{Outcome Measures}

The primary outcomes measures were the Bodily Pain and Physical Function scales of the Medical Outcomes Study 36item Short-Form General Health Survey (SF-36) and the modified Oswestry Disability Index at 6 weeks, 3 months, 6 months, 1 year, and 2 years. Secondary outcome measures included the SpS bothersomeness index, leg pain bothersomeness, low back pain bothersomeness, and patient satisfaction.

\section{Statistical Analysis}

Primary analyses compared surgical and nonoperative treatments using changes from baseline at each follow-up, with a mixed effects longitudinal regression model including a random individual effect to account for correlation between repeated measurements within individuals. Because of the crossover, analyses were based on treatments actually received in the combined randomized and observational cohorts. In these astreated analyses, the treatment indicator was a time-varying covariate, allowing for variable times of surgery. Times are measured from the beginning of treatment, i.e., the time of surgery for the surgical group and the time of enrollment for the nonoperative group. Therefore, all changes from baseline before surgery were included in the estimates of the nonoperative treatment effect. After surgery, changes were assigned to the surgical group with follow-up measured from the date of surgery. Repeated measures of outcomes were used as the dependent variables, and treatment received was included as a time-varying covariate. Adjustments were made for the time of surgery with respect to the original enrollment date so as to approximate the designated follow-up times. To adjust for potential confounding, baseline variables associated with missing data or treatment received were included as adjusting covariates in longitudinal regression models. ${ }^{12}$ The outcomes were stratified by the number of stenotic levels and outcomes between these subgroups were compared using a Wald test. Computations were done using SAS procedures PROC MIXED for continuous data and PROC GENMOD for binary and nonnormal secondary outcomes (SAS version 9.1 Windows XP Pro, Cary, NC). Statistical significance was defined as $P<0.05$ based on a 2-sided hypothesis test with no adjustments made for multiple comparisons.

\section{Results}

Spinal Stenosis Group. Analysis of the demographics of patients with 1,2 , or 3 or more levels of SpS, demonstrated several differences (Table 1). Patients with 3 or more levels of $\mathrm{SpS}$ were significantly older and more likely to be male. They were somewhat more likely to report comorbid hypertension and joint problems; they were more likely to report neurogenic claudication and less likely to have a radicular distribution of their pain. Single level SpS patients were more likely to smoke and suffer from depression. Work status did not differ between the groups nor did patient preference for mode of treatment. Radiographically, with increasing number of SpS levels, the likelihood that at least one level would be rated severe increased. The majority of patients with 1 
Table 1. Baseline Demographic Characteristics and Comorbidities According to Stenosis Level

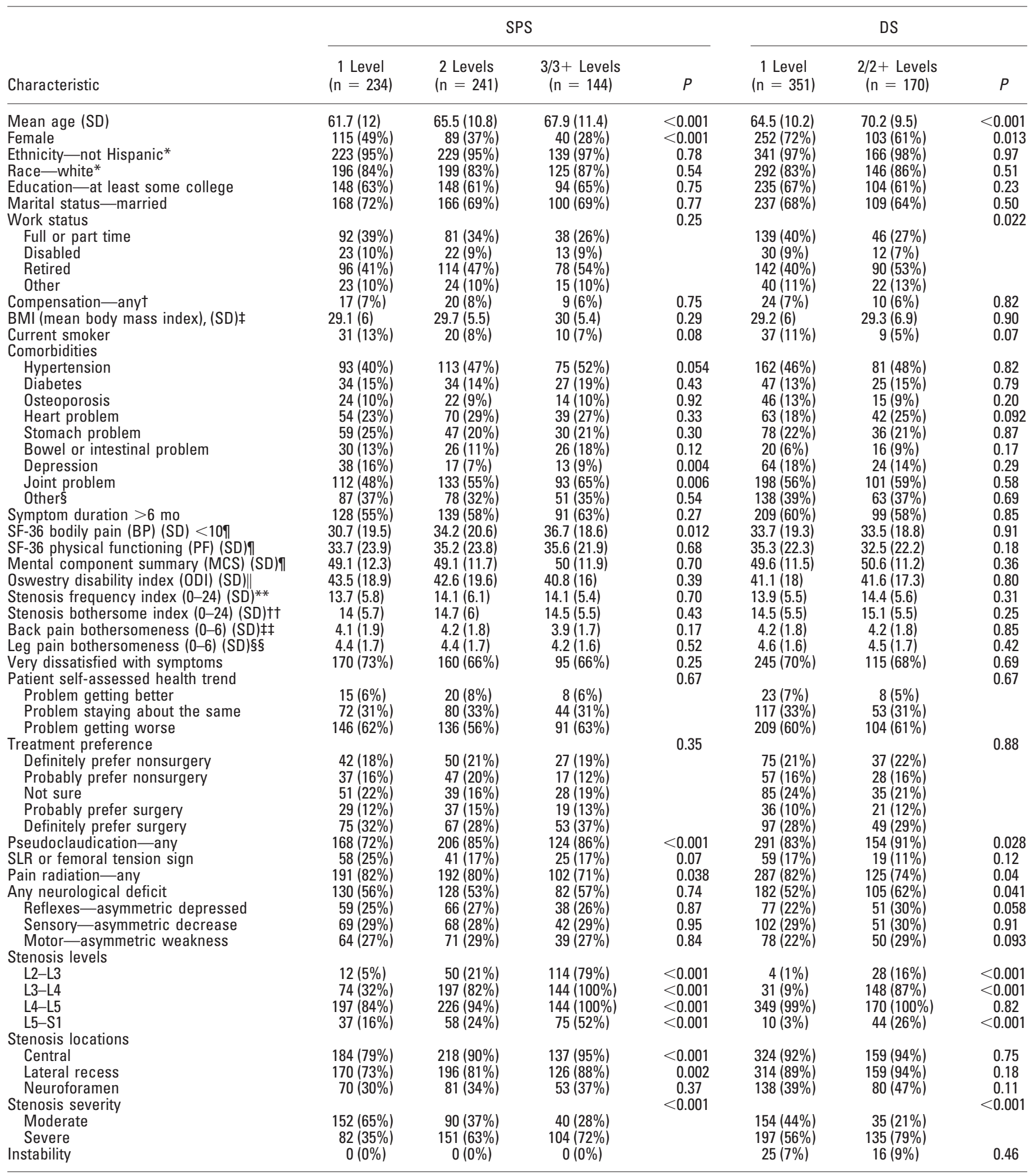

* Race or ethnic group was self-assessed. Whites and blacks could be either Hispanic or non-Hispanic.

tThis category includes patients who were receiving or had applications pending for workers compensation, social security, or other compensation.

₹The body-mass index is the weight in kilograms divided by the square of the height in meters.

$\S$ Other = problems related to stroke, cancer, lung, fibromyalgia, chronic fatigue syndrome, post traumatic stress disorder, alcohol, drug dependency, liver, kidney, blood vessel, nervous system, migraine, anxiety.

१The SF-36 scores range from 0 to 100, with higher score indicating less severe symptoms.

$\|$ The Oswestry Disability Index ranges from 1 to 100, with lower scores indicating less severe symptoms.

**The Stenosis Frequency Index ranges from 0 to 24 , with lower scores indicating less severe symptoms.

$\dagger+$ The Stenosis Bothersomeness Index ranges from 0 to 24 , with lower scores indicating less severe symptoms.

$\ddagger \ddagger$ The Low Back Pain Bothersomeness ranges from 0 to 6 , with lower scores indicating less severe symptoms.

$\S \S$ The Leg Pain Bothersomeness ranges from 0 to 6 , with lower scores indicating less severe symptoms.

$\mathrm{SpS}$ indicates spinal stenosis; DS, degenerative spondylolisthesis. 
level SpS had the L4-L5 level involved, 2 levels L3-L5, and 3 levels L2-L5.

Degenerative Spondylolisthesis Group. Similar to the $\mathrm{SpS}$ group, patients with multilevel SpS and DS were older, somewhat more likely to be male, less likely to smoke, and demonstrated more evidence of pseudoclaudication and less radiating pain. In contrast, however, in the DS cohort, multilevel SpS patients were more likely to be retired and clinically demonstrate asymmetric reflexes. Furthermore, no differences were found in medical comorbidities. Radiographically, the most common level of SpS was at L4-L5; multilevel SpS patients demonstrated severe radiographic signs of $\mathrm{SpS}$ similar to the $\mathrm{SpS}$ cohort (Table 1).

\section{Baseline Symptoms}

Spinal Stenosis Group. Patients with 3 or more levels of SpS had somewhat less severe pain at baseline on the SF-36 bodily pain scale compared to 1 and 2 levels (Table $1)$. Patients with single level $\mathrm{SpS}$ were less likely to present with neurogenic claudication $(P<0.001)$ and more likely to dermatomal pain radiation $(P=0.04)$. Other baseline symptoms were similar across groups.

Degenerative Spondylolisthesis Group. Patients did not demonstrate any statistical difference in baseline pain or disability scores (Table 1). Similar to the SpS group, those with single level stenosis were less likely to present with neurogenic claudication $(P=0.03)$ and more likely to have dermatomal pain radiation $(P=0.04)$. Patients with multilevel had a somewhat higher incidence of neurologic defects at baseline $(P=0.04)$. Other baseline symptoms were similar across groups.

\section{Surgical Procedures}

Spinal Stenosis Group. Overall, few patient underwent fusion in addition to their laminectomy and less so with increasing levels involved. With 3 or more levels of SpS, there were fewer instrumented fusions performed $(2 \%$ compared to $9 \%$ for 1 level) and no multilevel fusions compared to $5 \%$ in those with 1 or 2 levels. Operative time and intraoperative blood loss increased with increasing levels involved. Complications however, did not differ significantly nor did the rate of reoperations (Table 2). Mortality was extremely low and did not differ between subgroups.

Degenerative Spondylolisthesis Group. In comparison to the SpS group, the majority of patients in the spondylolisthesis group underwent a fusion with a trend toward noninstrumented fusion for increasing levels of SpS. Operative time did not differ significantly; however, blood loss increased with more levels. There was an overall trend towards more complications in the subgroup with multilevel stenosis as well (Table 2).

\section{Outcomes}

Spinal Stenosis Group. For all outcome measures, there were no differences in nonoperative outcomes when comparing 1, 2, or 3 or more level $\mathrm{SpS}$ at all time points except patient satisfaction at 2 years. More patients with 1 level SpS were satisfied with their symptoms than were patients with 2 or more levels. Interestingly, this difference was not seen at 1 year or in the percent of patients reporting major improvement at either 1 or 2 years (Table 3 ).

Surgical outcomes did not differ significantly at the various time points when comparing 1,2, or 3 level isolated SpS. The SF-36 physical function score was significantly better in the multilevel SpS group only at the 1-year assessment. This difference was not seen at 3 months or 2 years, or in any of the other measures (Table 3).

When comparing surgical to nonoperative treatments for 1, 2, or 3 level isolated SpS, there was a significant surgical treatment effect in most outcomes measures within each subgroup at each time point (Table 3). The only significant difference in treatment effects between subgroups was at 2 years for patient satisfaction with symptoms. Patients with single level SpS had a smaller difference in satisfaction between surgery and nonoperative treatment (i.e., a smaller treatment effect) than the other 2 groups.

Degenerative Spondylolisthesis Group. For the majority of outcome measures, there was no difference in nonoperative outcomes when comparing 1 or multiple level SpS with concomitant DS at the early time points. However, at 2 years, the SF-36 Bodily Pain and Physical Function scales, as well as the Oswestry Disability Index showed greater improvements in the single level patients compared to those with multilevel SpS. (Table 4).

The effect of multilevel involvement was even more pronounced in the surgical treatment group. The surgical outcomes were significantly better at 2 years in the single level patients compared to those with multilevel SpS for all primary outcome measures. Furthermore, SF-36 bodily pain and Oswestry scores remained significant at all time points, and at 1 year the self rated progress was also greater in those with single level SpS (Table 4).

As in the $\mathrm{SpS}$ group, surgical treatments demonstrated significant treatment improvement over nonoperative measures within each subgroup of DS patients (Table 4). The only significant difference in treatment effects between subgroups, however, was the low back pain bothersomeness at 2 years which showed a significantly greater advantage for surgery relative to nonoperative treatment among patients with single level SpS compared with their multiple level counterparts. At 3 months, SF-36 bodily pain and Oswestry Disability Index showed a trend favoring a greater surgical treatment effect for single level patients compared to multilevel patients.

\section{- Discussion}

SpS with and without associated degenerative spondylolisthesis is a common problem, and treatment choices are either nonoperative methods or surgical intervention. Various studies have compared these treat- 
Table 2. Operative Treatments, Complications and Events According to Stenosis Level

\begin{tabular}{|c|c|c|c|c|c|c|c|}
\hline \multirow[b]{2}{*}{ Characteristic } & \multicolumn{4}{|c|}{ SPS } & \multicolumn{3}{|c|}{ DS } \\
\hline & $\begin{array}{l}1 \text { Level } \\
(n=139)\end{array}$ & $\begin{array}{l}2 \text { Levels } \\
(\mathrm{n}=154)\end{array}$ & $\begin{array}{l}3 / 3+\text { Levels } \\
(n=95)\end{array}$ & $P$ & $\begin{array}{l}1 \text { Level } \\
(n=224)\end{array}$ & $\begin{array}{c}2 / 2+\text { Levels } \\
(n=104)\end{array}$ & $P$ \\
\hline Specific procedures & & & & 0.046 & & & 0.092 \\
\hline Decompression only & $121(88 \%)$ & $134(89 \%)$ & $86(91 \%)$ & & $9(4 \%)$ & $7(7 \%)$ & \\
\hline Non-instrumented fusion & $3(2 \%)$ & $10(7 \%)$ & $7(7 \%)$ & & $43(19 \%)$ & $29(28 \%)$ & \\
\hline Instrumented fusion & $13(9 \%)$ & $7(5 \%)$ & $2(2 \%)$ & & $172(77 \%)$ & $68(65 \%)$ & \\
\hline Multilevel fusion & $7(5 \%)$ & $8(5 \%)$ & $0(0 \%)$ & 0.079 & $45(20 \%)$ & $30(29 \%)$ & 0.11 \\
\hline \multicolumn{8}{|l|}{ Decompression level } \\
\hline $\mathrm{L} 2-\mathrm{L} 3$ & $14(10 \%)$ & $54(35 \%)$ & $75(79 \%)$ & $<0.001$ & $6(3 \%)$ & $26(25 \%)$ & $<0.001$ \\
\hline L3-L4 & $55(41 \%)$ & $124(81 \%)$ & $91(96 \%)$ & $<0.001$ & $61(28 \%)$ & $83(80 \%)$ & $<0.001$ \\
\hline L4-L5 & $119(89 \%)$ & $143(93 \%)$ & $91(96 \%)$ & 0.12 & $223(100 \%)$ & $102(98 \%)$ & 0.49 \\
\hline L5-S1 & $39(29 \%)$ & $62(41 \%)$ & $48(51 \%)$ & 0.004 & $53(24 \%)$ & $42(40 \%)$ & 0.004 \\
\hline Levels decompressed & & & & $<0.001$ & & & $<0.001$ \\
\hline None & $5(4 \%)$ & $1(1 \%)$ & $0(0 \%)$ & & $0(0 \%)$ & $0(0 \%)$ & \\
\hline One & $68(49 \%)$ & $14(9 \%)$ & $3(3 \%)$ & & $131(58 \%)$ & $12(12 \%)$ & \\
\hline Two & $45(32 \%)$ & $65(42 \%)$ & $11(12 \%)$ & & $69(31 \%)$ & $48(46 \%)$ & \\
\hline Three+ & $21(15 \%)$ & $74(48 \%)$ & $81(85 \%)$ & & $24(11 \%)$ & $44(42 \%)$ & \\
\hline Operation time, minutes (SD) & $114.9(71.7)$ & $128.8(57.1)$ & $146.2(63.9)$ & 0.001 & $208.4(86.7)$ & $204.8(78)$ & 0.72 \\
\hline Blood loss, cc (SD) & $221(444.1)$ & $338.1(384.6)$ & $415.3(381.3)$ & 0.001 & $532.3(393.7)$ & $732.4(605.6)$ & $<0.001$ \\
\hline \multicolumn{8}{|l|}{ Blood replacement } \\
\hline Intraoperative replacement & $12(9 \%)$ & $19(12 \%)$ & $6(6 \%)$ & 0.25 & $74(33 \%)$ & $42(40 \%)$ & 0.26 \\
\hline Post-operative transfusion & $7(5 \%)$ & $7(5 \%)$ & $5(5 \%)$ & 0.97 & $43(19 \%)$ & $24(23 \%)$ & 0.53 \\
\hline Length of hospital stay (SD) & $2.8(2.5)$ & $5.7(30)$ & $3.6(3)$ & 0.40 & $6.4(24.9)$ & $4.8(3.2)$ & 0.52 \\
\hline \multicolumn{8}{|l|}{ Intraoperative complications* } \\
\hline Dural tear/spinal fluid leak & $8(6 \%)$ & $19(12 \%)$ & $9(9 \%)$ & 0.15 & $19(8 \%)$ & $14(13 \%)$ & 0.23 \\
\hline Vascular injury & $0(0 \%)$ & $0(0 \%)$ & $0(0 \%)$ & & $0(0 \%)$ & $1(1 \%)$ & 0.69 \\
\hline Other & $1(1 \%)$ & $1(1 \%)$ & $1(1 \%)$ & 0.94 & $7(3 \%)$ & $2(2 \%)$ & 0.80 \\
\hline None & $130(94 \%)$ & $133(87 \%)$ & $85(89 \%)$ & 0.17 & $199(89 \%)$ & $88(85 \%)$ & 0.37 \\
\hline \multicolumn{8}{|l|}{ Postoperative complications/events $\dagger$} \\
\hline Nerve root injury & $0(0 \%)$ & $0(0 \%)$ & $0(0 \%)$ & & $0(0 \%)$ & $1(1 \%)$ & 0.70 \\
\hline Wound dehiscence & $0(0 \%)$ & $0(0 \%)$ & $0(0 \%)$ & & $1(0 \%)$ & $0(0 \%)$ & 0.70 \\
\hline Wound hematoma & $1(1 \%)$ & $1(1 \%)$ & $2(2 \%)$ & 0.50 & $1(0 \%)$ & $0(0 \%)$ & 0.70 \\
\hline Wound Infection & $4(3 \%)$ & $2(1 \%)$ & $1(1 \%)$ & 0.49 & $10(5 \%)$ & $1(1 \%)$ & 0.19 \\
\hline Other & $5(4 \%)$ & $10(7 \%)$ & $6(6 \%)$ & 0.49 & $16(7 \%)$ & $15(14 \%)$ & 0.062 \\
\hline None & $122(88 \%)$ & $134(88 \%)$ & $82(86 \%)$ & 0.88 & $157(71 \%)$ & $68(65 \%)$ & 0.40 \\
\hline $\begin{array}{l}\text { Postoperative mortality (death within } \\
6 \text { wk of surgery) }\end{array}$ & $1 \mp(0.7 \%)$ & $0(0 \%)$ & $0(0 \%)$ & 0.41 & $1(0.4 \%)$ & $0(0 \%)$ & 0.69 \\
\hline $\begin{array}{l}\text { Post-operative mortality (death } \\
\text { within } 3 \text { mo of surgery) }\end{array}$ & $1 \ddagger(0.7 \%)$ & $0(0 \%)$ & $0(0 \%)$ & 0.41 & $1(0.4 \%)$ & $1(1 \%)$ & 0.84 \\
\hline Additional surgeries (1-yr rate)‡ & $6(4 \%)$ & $11(7 \%)$ & $3(3 \%)$ & 0.316 & $16(7 \%)$ & $5(5 \%)$ & 0.41 \\
\hline Additional surgeries (2-yr rate)‡ & $10(7 \%)$ & $15(10 \%)$ & $5(5 \%)$ & 0.402 & $24(11 \%)$ & $18(17 \%)$ & 0.127 \\
\hline $\begin{array}{l}\text { Recurrent stenosis/progressive } \\
\text { listhesis }\end{array}$ & $4(2.8 \%)$ & $4(2.6 \%)$ & $2(2.1 \%)$ & & $4(1.8 \%)$ & $7(6.8 \%)$ & \\
\hline Pseudarthrosis/fusion exploration & $0(0 \%)$ & $0(0 \%)$ & $0(0 \%)$ & & $2(0.9 \%)$ & $1(1 \%)$ & \\
\hline Complication or other & $5(3.5 \%)$ & $9(5.9 \%)$ & $0(0 \%)$ & & $17(7.6 \%)$ & $5(4.9 \%)$ & \\
\hline New condition & $1(0.7 \%)$ & $3(2 \%)$ & $2(2.1 \%)$ & & $2(0.9 \%)$ & $3(2.9 \%)$ & \\
\hline
\end{tabular}

*None of the following were reported: aspiration, nerve root injury, operation at wrong level.

tAny reported complications up to 8-wk postoperation. None of the following were reported: bone graft complication, CSF leak, paralysis, cauda equina injury, pseudarthrosis.

¥1- and 2-yr postsurgical reoperation rates are Kaplan Meier estimates and P-values are based on log-rank test.

SpS indicates spinal stenosis; DS, degenerative spondylolisthesis.

ments choices, ${ }^{6-9,13}$ the most recent being the SPORT study. ${ }^{10,11}$ These studies demonstrated significant benefit from surgical intervention initially but a decline in benefit with time. In particular, the SPORT study demonstrated, in the as-treated analysis, the treatment effect for surgery was seen as early as 6 weeks, appeared to reach a maximum at 6 months, and persisted for 2 years. In comparison, the nonoperative patients improved only moderately during the 2 -year period. ${ }^{10,11}$ In this subanalysis of SPORT data, multilevel SpS did not demonstrate worse baseline symptoms or worse treatment outcomes in isolated SpS; however, if concomitant DS existed, single level patients tended to improve more than multilevel patients, particularly with surgery.
Despite the hypothesis that multilevel $\mathrm{SpS}$ patients fair worse with nonoperative treatment, this study did not find this to be true in patients with isolated $\mathrm{SpS}$ and normal alignment. Nonoperative treatment ranged from bed rest, NSAIDS, analgesics, physical therapy, and oral corticosteroids. Nonoperative treatment has been suggested to improve outcomes through improved lumbopelvic muscular stabilization enabling better maintenance of posterior pelvic tilt. ${ }^{14}$ Improved cardiovascular conditioning has also been suggested as having a positive treatment effect. ${ }^{14}$ If these theories are true, multilevel SpS should not inhibit a patient's ability to improve their symptomology. In a study by Simotas et al, 49 patients underwent nonoperative treatment. ${ }^{14}$ At 3 years, 9 of the 
Table 3. Subgroup Results From Common Adjusting Covariates* As-Treated Outcome Analysis by Number of Levels With Moderate or Severe Stenosis for the Randomized and Observational SPS Cohorts Combined

\begin{tabular}{|c|c|c|c|c|c|c|c|c|c|c|}
\hline \multirow[b]{2}{*}{$\begin{array}{l}\text { SPS Cohort } \\
\text { Outcomes }\end{array}$} & \multirow[b]{2}{*}{$\begin{array}{l}\text { Stenosis } \\
\text { Level }\end{array}$} & \multicolumn{3}{|c|}{ 3-mo } & \multicolumn{3}{|c|}{$1-y r$} & \multicolumn{3}{|c|}{$2-y r$} \\
\hline & & Surgical & Non-Operative & $\begin{array}{l}\text { Treatment Effect } \\
\qquad(95 \% \mathrm{Cl})\end{array}$ & Surgical & Non-Operative & $\begin{array}{l}\text { Treatment Effect } \\
\qquad(95 \% \mathrm{CI})\end{array}$ & Surgical & Non-Operative & $\begin{array}{l}\text { Treatment Effect } † \\
\qquad(95 \% \mathrm{Cl})\end{array}$ \\
\hline \multirow{3}{*}{$\begin{array}{l}\text { SF-36 bodily pain } \\
\text { (BP) (0-100) } \\
\text { (SE) } \ddagger\end{array}$} & One & $31(2)$ & $13.2(2.1)$ & $17.8(12.8,22.9)$ & $29.6(2.2)$ & $16.8(2.4)$ & $12.8(7,18.6)$ & $28.6(2.1)$ & $17.3(2.5)$ & $11.2(5.2,17.3)$ \\
\hline & Two & $27.9(1.9)$ & $12(2)$ & $15.9(11,20.8)$ & $27.6(2.1)$ & $11.5(2.4)$ & $16.1(10.3,21.9)$ & $27.9(2.1)$ & $12.8(2.5)$ & $15.1(9,21.1)$ \\
\hline & $\begin{array}{l}\text { Three+ } \\
P\end{array}$ & $\begin{array}{c}30.5(2.5) \\
0.49\end{array}$ & $\begin{array}{l}15(2.9) \\
0.69\end{array}$ & $\begin{array}{c}15.5(8.8,22.3) \\
0.81\end{array}$ & $\begin{array}{c}33.8(2.5) \\
0.16\end{array}$ & $\begin{array}{c}16.4(3.2) \\
0.23\end{array}$ & $\begin{array}{c}17.4(9.8,25.1) \\
0.58\end{array}$ & $\begin{array}{c}28.7(2.5) \\
0.96\end{array}$ & $\begin{array}{c}13.9(3.4) \\
0.43\end{array}$ & $\begin{array}{c}14.7(6.9,22.6) \\
0.63\end{array}$ \\
\hline \multirow{4}{*}{$\begin{array}{l}\text { SF-36 physical } \\
\text { function (PF) } \\
(0-100)(S E) \ddagger\end{array}$} & One & $25.3(1.9)$ & $11.7(2)$ & $13.7(9,18.3)$ & $26.4(2.1)$ & $12.9(2.2)$ & $13.5(8.1,18.9)$ & $23(2)$ & $13.7(2.4)$ & $9.3(3.7,15)$ \\
\hline & Two & $22.7(1.8)$ & $8.1(1.9)$ & $14.6(10,19.1)$ & $23.2(2)$ & $9.3(2.2)$ & $13.9(8.6,19.3)$ & $21.5(2)$ & $10.5(2.4)$ & $11(5.4,16.6)$ \\
\hline & Three+ & $26.8(2.4)$ & $10.5(2.7)$ & $16.4(10.1,22.6)$ & $30.6(2.4)$ & $9.4(3.1)$ & $21.3(14.1,28.4)$ & $25.2(2.4)$ & $10.4(3.2)$ & $14.7(7.4,22.1)$ \\
\hline & $P$ & 0.34 & 0.42 & 0.79 & 0.057 & 0.47 & 0.18 & 0.48 & 0.59 & 0.51 \\
\hline \multirow{4}{*}{$\begin{array}{l}\text { Mental component } \\
\text { summary (MCS) } \\
(0-100) \text { (SE) } \ddagger\end{array}$} & One & $3.9(0.8)$ & $2.2(0.9)$ & $1.7(-0.5,3.8)$ & $2.8(0.9)$ & $2.6(1)$ & $0.2(-2.2,2.7)$ & $2.8(0.9)$ & $1.5(1)$ & $1.3(-1.3,3.9)$ \\
\hline & Two & $4(0.8)$ & $1.5(0.8)$ & $2.5(0.4,4.6)$ & $3.6(0.9)$ & $1.2(1)$ & $2.4(-0.1,4.8)$ & $4(0.9)$ & $1.7(1)$ & $2.2(-0.3,4.8)$ \\
\hline & Three+ & $4.2(1)$ & $2.4(1.2)$ & $1.8(-1.1,4.6)$ & $5.1(1)$ & $3.5(1.3)$ & $1.6(-1.6,4.8)$ & $4.7(1)$ & $-0.4(1.4)$ & $5.1(1.8,8.4)$ \\
\hline & $P$ & 0.98 & 0.79 & 0.84 & 0.27 & 0.34 & 0.46 & 0.35 & 0.43 & 0.19 \\
\hline \multirow{4}{*}{$\begin{array}{l}\text { Oswestry disability } \\
\text { index (ODI) } \\
(0-100)(\text { SE)§ }\end{array}$} & One & $-20.6(1.6)$ & $-8.9(1.6)$ & $-11.7(-15.5,-7.9)$ & $-19.9(1.7)$ & $-10.6(1.8)$ & $-9.3(-13.7,-4.9)$ & $-19.8(1.6)$ & $-10.4(1.9)$ & $-9.4(-14,-4.8)$ \\
\hline & Two & $-21.3(1.5)$ & $-5.1(1.6)$ & $-16.2(-19.9,-12.5)$ & $-20.6(1.6)$ & $-7.4(1.8)$ & $-13.2(-17.6,-8.8)$ & $-20.2(1.6)$ & $-9.4(1.9)$ & $-10.7(-15.3,-6.2)$ \\
\hline & Three+ & $-22.4(1.9)$ & $-9.6(2.2)$ & $-12.8(-17.9,-7.7)$ & $-24.4(2)$ & $-8.4(2.5)$ & $-16(-21.8,-10.2)$ & $-21.9(1.9)$ & $-6.3(2.6)$ & $-15.7(-21.6,-9.7)$ \\
\hline & $P$ & 0.78 & 0.12 & 0.21 & 0.19 & 0.46 & 0.17 & 0.68 & 0.45 & 0.25 \\
\hline \multirow{4}{*}{$\begin{array}{l}\text { Stenosis frequency } \\
\text { index (0-24) } \\
\text { (SE)ף }\end{array}$} & One & $-7.7(0.7)$ & $-3.3(0.6)$ & $-4.4(-6,-2.8)$ & $-7.3(0.6)$ & $-3.8(0.6)$ & $-3.4(-5,-1.8)$ & $-6.9(0.6)$ & $-4.4(0.7)$ & $-2.6(-4.3,-0.9)$ \\
\hline & Two & $-7.5(0.7)$ & $-2(0.6)$ & $-5.5(-7.2,-3.8)$ & $-7(0.6)$ & $-2.9(0.6)$ & $-4.1(-5.7,-2.5)$ & $-7.3(0.6)$ & $-3.9(0.7)$ & $-3.3(-5.1,-1.6)$ \\
\hline & Three+ & $-8.6(0.8)$ & $-3.5(0.8)$ & $-5(-7.2,-2.9)$ & $-8.3(0.7)$ & $-4.1(0.9)$ & $-4.2(-6.3,-2.1)$ & $-7.4(0.7)$ & $-2.8(0.9)$ & $-4.6(-6.8,-2.4)$ \\
\hline & $P$ & 0.60 & 0.13 & 0.65 & 0.30 & 0.43 & 0.79 & 0.85 & 0.43 & 0.35 \\
\hline \multirow{4}{*}{$\begin{array}{l}\text { Stenosis } \\
\quad \text { bothersomeness } \\
\text { index (0-24) (SE)ף }\end{array}$} & One & $-8.4(0.7)$ & $-3.4(0.6)$ & $-4.9(-6.6,-3.3)$ & $-8.2(0.6)$ & $-4(0.6)$ & $-4.2(-5.8,-2.6)$ & $-7.4(0.6)$ & $-4.5(0.7)$ & $-2.8(-4.5,-1.1)$ \\
\hline & Two & $-8.5(0.7)$ & $-2.2(0.6)$ & $-6.3(-8,-4.5)$ & $-7.7(0.6)$ & $-3.7(0.7)$ & $-4.1(-5.7,-2.4)$ & $-8.1(0.6)$ & $-4.3(0.7)$ & $-3.7(-5.5,-2)$ \\
\hline & Three+ & $-8.9(0.8)$ & $-3.2(0.8)$ & $-5.7(-7.8,-3.5)$ & $-9(0.7)$ & $-3.9(0.9)$ & $-5.2(-7.3,-3)$ & $-8(0.7)$ & $-4.1(0.9)$ & $-3.9(-6.1,-1.7)$ \\
\hline & $P$ & 0.89 & 0.27 & 0.53 & 0.33 & 0.95 & 0.68 & 0.66 & 0.92 & 0.66 \\
\hline \multirow{4}{*}{$\begin{array}{l}\text { Low back pain } \\
\text { bothersomeness } \\
(0-6)(\text { SE) } \|\end{array}$} & One & $-1.8(0.2)$ & $-0.8(0.2)$ & $-1(-1.5,-0.5)$ & $-2(0.2)$ & $-1(0.2)$ & $-1(-1.5,-0.5)$ & $-2.1(0.2)$ & $-1.3(0.2)$ & $-0.8(-1.3,-0.3)$ \\
\hline & Two & $-2.2(0.2)$ & $-0.8(0.2)$ & $-1.4(-1.9,-0.9)$ & $-1.9(0.2)$ & $-1.1(0.2)$ & $-0.8(-1.3,-0.3)$ & $-1.9(0.2)$ & $-1.1(0.2)$ & $-0.8(-1.3,-0.3)$ \\
\hline & Three+ & $-2(0.2)$ & $-1(0.2)$ & $-1(-1.6,-0.4)$ & $-2.2(0.2)$ & $-1(0.3)$ & $-1.2(-1.8,-0.6)$ & $-2.1(0.2)$ & $-0.8(0.3)$ & $-1.3(-1.9,-0.7)$ \\
\hline & $P$ & 0.42 & 0.64 & 0.43 & 0.39 & 0.94 & 0.58 & 0.75 & 0.28 & 0.35 \\
\hline \multirow{4}{*}{$\begin{array}{l}\text { Very/somewhat } \\
\text { satisfied with } \\
\text { symptoms (\%) }\end{array}$} & One & 62.1 & 24.7 & $37.4(26.1,48.7)$ & 67.3 & 28.9 & $38.4(25.4,51.4)$ & 63.8 & 44.7 & $19.1(4.3,33.9)$ \\
\hline & Two & 63.2 & 21.7 & $41.5(30.8,52.2)$ & 62.3 & 29.7 & $32.6(19.3,45.8)$ & 71.5 & 22 & $49.5(37,62)$ \\
\hline & Three+ & 56.1 & 24.9 & $31.2(16.3,46.1)$ & 75.3 & 32.1 & $43.2(26.7,59.7)$ & 69 & 21.2 & $47.8(32,63.6)$ \\
\hline & $P$ & 0.59 & 0.83 & 0.57 & 0.19 & 0.93 & 0.61 & 0.48 & 0.01 & 0.005 \\
\hline \multirow{4}{*}{$\begin{array}{l}\text { Self-rated progress } \\
\text { major improvement } \\
(\%)\end{array}$} & One & 72.4 & 23.9 & $48.4(38,58.9)$ & 65.9 & 27.6 & $38.3(25.6,51)$ & 62.3 & 36.2 & $26.1(12.1,40.2)$ \\
\hline & Two & 70.4 & 17.6 & $52.8(43.2,62.4)$ & 66.1 & 24.8 & $41.3(29,53.7)$ & 64.7 & 25.2 & $39.5(26.5,52.5)$ \\
\hline & Three+ & 71.6 & 17.3 & $54.2(41.4,67.1)$ & 74.6 & 26.4 & $48.2(32.7,63.8)$ & 59.4 & 24.5 & $34.9(18.2,51.6)$ \\
\hline & $P$ & 0.95 & 0.43 & 0.70 & 0.39 & 0.92 & 0.63 & 0.77 & 0.30 & 0.42 \\
\hline
\end{tabular}

*Adjusted for age, gender, stomach comorbidity, SLR or femoral tension sign, smoking status, joint comorbidity, patient self assessed health trend, income, compensation, BMI, baseline score (for SF-36 and ODI), and center.

tTreatment effect is the difference between the surgical and non-operative mean change from baseline. Analysis is done using a mixed model with a random subject intercept term. Treatment is a time-varying covariate where a patients' experience prior to surgery is attributed to the nonoperative arm and time is measured from enrollment and his/her post-surgery outcomes are attributed to the surgical arm and time is measured from time of surgery.

¥The SF-36 scores range from 0 to 100, with higher score indicating less severe symptoms.

$\S$ The Oswestry Disability Index ranges from 1 to 100, with lower scores indicating less severe symptoms.

IThe Stenosis Bothersomeness Index and the Stenosis Frequency Index range from 0 to 24, with lower scores indicating less severe symptoms.

\|The Low Back Pain Bothersomeness Scale ranges from 0 to 6, with lower scores indicating less severe symptoms.

49 had surgical intervention and of the remaining 40 patients, 2 suffered significant motor deterioration, 5 worse, 12 no change, 11 mild improvement, and 12 sustained improvement. This study found a trend between worse outcomes and more levels of SpS, using patient reported scales such as visual analog scales, RolandMorris disability scale, and overall rating of anxiety and depression. ${ }^{14}$ Importantly, the authors stated more patients needed to be analyzed to see if this conclusion is valid. Furthermore, this study population had a mixture of SpS cases with and without DS. ${ }^{14}$ Those significant differences may have been due to the DS patients.

Contrary to Simotas et al, the other few studies that mention the effect of multilevel SpS and outcome do not demonstrate a difference in number of SpS levels and outcomes. These studies unfortunately included patients with and without DS. Amundsen et al stated patients with multilevel afflictions, treated surgically or nonoperatively, did not have poorer outcome than those with single level SpS. ${ }^{9}$ Yukawa et al demonstrated when operating in cases with single versus 2 level SpS, outcomes did not change as measured by the Oswestry disability index and a visual analog scale. ${ }^{15}$ They suggest that multilevel SpS should not affect surgical outcome if each compressed level is adequately addressed at surgery. ${ }^{15}$

Although this subanalysis demonstrated no significant difference in surgical outcomes in patients with isolated SpS similar to previous published reports, patients with DS and multiple levels of SpS demonstrated worse outcomes for all primary and secondary outcome measurements at 2 years. This difference suggests that these 2 disease entities differ in clinically important ways. In the pathogenesis of DS, secondary degeneration of the facets, ligamentum flavum, and osteophytic spurs develop because of the subtle instability of the spondylolisthesis segment. ${ }^{16,17}$ Compression of neural elements can be from both the slippage of the vertebral segment and secondary degeneration in contrast to only primary degen- 


\begin{abstract}
Table 4. Subgroup Results From Common Adjusting Covariates* As-Treated Outcome Analysis by Number of Levels With Moderate or Severe Stenosis for the Randomized and Observational Cohorts Combined DS Cohort With Listhesis Level of L4-L5
\end{abstract}

\begin{tabular}{|c|c|c|c|c|c|c|c|c|c|c|}
\hline \multirow[b]{2}{*}{$\begin{array}{l}\text { DS Cohort } \\
\text { Outcomes }\end{array}$} & \multirow[b]{2}{*}{$\begin{array}{l}\text { Stenosis } \\
\text { Level }\end{array}$} & \multicolumn{3}{|c|}{ 3-mo } & \multicolumn{3}{|c|}{$1-y r$} & \multicolumn{3}{|c|}{$2-y r$} \\
\hline & & Surgical & Non-Operative & $\begin{array}{l}\text { Treatment Effect } \dagger \\
\qquad(95 \% \mathrm{Cl})\end{array}$ & Surgical & Non-Operative & $\begin{array}{l}\text { Treatment Effect } \dagger \\
\qquad(95 \% \mathrm{Cl})\end{array}$ & Surgical & Non-Operative & $\begin{array}{l}\text { Treatment Effect } \dagger \\
(95 \% \mathrm{CI})\end{array}$ \\
\hline \multirow{3}{*}{$\begin{array}{l}\text { SF-36 bodily pain } \\
\text { (BP) (0-100) } \\
\text { (SE) } \ddagger\end{array}$} & One & $32.7(1.5)$ & $11.6(1.7)$ & $21.2(17.2,25.1)$ & $34.9(1.6)$ & $13.9(1.9)$ & $21(16.5,25.6)$ & $34.9(1.6)$ & $16(2)$ & $18.8(14.1,23.6)$ \\
\hline & Two/three+ & $24.4(2.2)$ & $9.5(2.4)$ & $14.9(9.3,20.5)$ & $27(2.4)$ & $9.8(2.7)$ & $17.2(10.6,23.9)$ & $22.8(2.4)$ & $8.9(2.9)$ & $13.9(6.9,20.9)$ \\
\hline & $P$ & 0.002 & 0.48 & 0.066 & 0.008 & 0.22 & 0.35 & $<0.001$ & 0.045 & 0.24 \\
\hline \multirow{2}{*}{$\begin{array}{l}\text { SF-36 physical } \\
\text { function (PF) }\end{array}$} & One & $23.1(1.5)$ & $7.4(1.6)$ & $15.7(12,19.5)$ & $29.7(1.5)$ & $9(1.8)$ & $20.7(16.4,25.1)$ & $28.5(1.5)$ & $10.8(1.9)$ & $17.7(13.1,22.2)$ \\
\hline & Two/three+ & $19.3(2.1)$ & $9(2.3)$ & $10.3(5,15.6)$ & $28.3(2.4)$ & $8.3(2.6)$ & $19.9(13.6,26.2)$ & $21.3(2.3)$ & $2.6(2.8)$ & $18.7(12,25.3)$ \\
\hline$(0-100)(S E) \ddagger$ & $P$ & 0.14 & 0.56 & 0.092 & 0.62 & 0.84 & 0.84 & 0.007 & 0.016 & 0.80 \\
\hline \multirow{2}{*}{$\begin{array}{r}\text { Mental component } \\
\text { summary (MCS) }\end{array}$} & One & $4.4(0.6)$ & $2.1(0.7)$ & $2.3(0.6,4)$ & $3.4(0.7)$ & $0.8(0.8)$ & $2.5(0.6,4.5)$ & $3.3(0.7)$ & $1.1(0.9)$ & $2.1(0.1,4.2)$ \\
\hline & Two/three+ & $3.2(0.9)$ & $0.3(1)$ & $2.9(0.5,5.3)$ & $2.9(1)$ & $2.4(1.1)$ & $0.5(-2.4,3.4)$ & $1.7(1)$ & $-0.3(1.2)$ & $2(-1,5.1)$ \\
\hline$(0-100)$ (SE) $\ddagger$ & $P$ & 0.27 & 0.13 & 0.68 & 0.73 & 0.25 & 0.25 & 0.20 & 0.35 & 0.96 \\
\hline \multirow{3}{*}{$\begin{array}{l}\text { Oswestry disability } \\
\text { index (ODI) (0- } \\
\text { 100) (SE)§ }\end{array}$} & One & $-22(1.2)$ & $-5.8(1.3)$ & $-16.2(-19.1,-13.2)$ & $-26.4(1.2)$ & $-7.8(1.4)$ & $-18.5(-21.9,-15.1)$ & $-25.6(1.2)$ & $-9.1(1.5)$ & $-16.5(-20.1,-12.9)$ \\
\hline & Two/three+ & $-18.4(1.7)$ & $-6.6(1.8)$ & $-11.8(-16,-7.7)$ & $-22.1(1.9)$ & $-3.9(2.1)$ & $-18.2(-23.2,-13.2)$ & $-19.5(1.8)$ & $-2.7(2.3)$ & $-16.7(-22,-11.4)$ \\
\hline & $P$ & 0.085 & 0.72 & 0.088 & 0.062 & 0.13 & 0.91 & 0.005 & 0.021 & 0.94 \\
\hline \multirow{3}{*}{$\begin{array}{l}\text { Stenosis } \\
\text { frequency index } \\
(0-24)(S E) \uparrow\end{array}$} & One & $-9.5(0.6)$ & $-1.9(0.4)$ & $-7.6(-8.9,-6.2)$ & $-9.2(0.4)$ & $-2.9(0.5)$ & $-6.3(-7.5,-5.1)$ & $-8.7(0.4)$ & $-3.8(0.6)$ & $-4.9(-6.2,-3.6)$ \\
\hline & Two/three+ & $-9.1(0.9)$ & $-3.4(0.6)$ & $-5.7(-7.7,-3.7)$ & $-7.9(0.7)$ & $-2.6(0.7)$ & $-5.3(-7.1,-3.4)$ & $-6.6(0.7)$ & $-2.8(0.8)$ & $-3.8(-5.7,-1.9)$ \\
\hline & $P$ & 0.71 & 0.059 & 0.12 & 0.10 & 0.76 & 0.35 & 0.006 & 0.33 & 0.32 \\
\hline \multirow{3}{*}{$\begin{array}{l}\text { Stenosis } \\
\quad \text { bothersomeness } \\
\text { index (0-24) } \\
\text { (SE)ף }\end{array}$} & One & $-10.7(0.6)$ & $-2.6(0.5)$ & $-8.1(-9.5,-6.7)$ & $-9.9(0.4)$ & $-3.3(0.5)$ & $-6.6(-7.9,-5.3)$ & $-9.5(0.4)$ & $-4(0.6)$ & $-5.5(-6.9,-4.2)$ \\
\hline & Two/three+ & $-9.6(0.9)$ & $-3.5(0.7)$ & $-6.2(-8.2,-4.1)$ & $-8.7(0.7)$ & $-3.7(0.8)$ & $-4.9(-6.8,-3)$ & $-8(0.7)$ & $-3.6(0.8)$ & $-4.4(-6.4,-2.4)$ \\
\hline & $P$ & 0.32 & 0.29 & 0.13 & 0.13 & 0.65 & 0.15 & 0.059 & 0.74 & 0.34 \\
\hline \multirow{3}{*}{$\begin{array}{l}\text { Low back pain } \\
\text { bothersomeness } \\
(0-6)(S E) \|\end{array}$} & One & $-2.3(0.2)$ & $-1(0.1)$ & $-1.3(-1.7,-0.9)$ & $-2.5(0.1)$ & $-0.9(0.1)$ & $-1.5(-1.9,-1.1)$ & $-2.2(0.1)$ & $-1.1(0.2)$ & $-1.2(-1.6,-0.8)$ \\
\hline & Two/three+ & $-2.2(0.3)$ & $-1.2(0.2)$ & $-1(-1.6,-0.4)$ & $-2.2(0.2)$ & $-1.2(0.2)$ & $-1(-1.5,-0.5)$ & $-1.7(0.2)$ & $-1.3(0.2)$ & $-0.5(-1.1,0.1)$ \\
\hline & $P$ & 0.76 & 0.41 & 0.46 & 0.20 & 0.31 & 0.11 & 0.027 & 0.45 & 0.043 \\
\hline \multirow{3}{*}{$\begin{array}{l}\text { Very/somewhat } \\
\text { satisfied with } \\
\text { symptoms (\%) }\end{array}$} & One & 72.2 & 24.9 & $47.3(38.8,55.9)$ & 75.3 & 29.3 & $46(36,56.1)$ & 72.3 & 37.2 & $35.2(24,46.3)$ \\
\hline & Two/three+ & 64.7 & 25.1 & $39.6(27,52.1)$ & 66.2 & 21.2 & $45(30.6,59.3)$ & 52.3 & 30.3 & $22.1(5.3,38.8)$ \\
\hline & $P$ & 0.23 & 0.97 & 0.35 & 0.17 & 0.23 & 0.98 & 0.003 & 0.44 & 0.25 \\
\hline \multirow{3}{*}{$\begin{array}{l}\text { Self-rated } \\
\text { progress major } \\
\text { improvement } \\
(\%)\end{array}$} & One & 79.6 & 21.3 & $58.3(50.4,66.1)$ & 80.5 & 26.2 & $54.4(45.1,63.7)$ & 77.1 & 27.9 & $49.2(39.1,59.4)$ \\
\hline & Two/three+ & 71.9 & 22 & $49.9(38.2,61.7)$ & 66.8 & 22.7 & $44.1(29.7,58.4)$ & 59.6 & 20.3 & $39.3(24.3,54.3)$ \\
\hline & $P$ & 0.17 & 0.91 & 0.28 & 0.024 & 0.62 & 0.25 & 0.005 & 0.35 & 0.43 \\
\hline
\end{tabular}

*Adjusted for age, gender, stomach comorbidity, SLR or femoral tension sign, smoking status, joint comorbidity, patient self assessed health trend, income, compensation, BMI, baseline score (for SF-36 and ODI), and center.

tTreatment effect is the difference between the surgical and non-operative mean change from baseline. Analysis is done using a mixed model with a random subject intercept term. Treatment is a time-varying covariate where a patients' experience prior to surgery is attributed to the non-operative arm and time is measured from enrollment and his/her post-surgery outcomes are attributed to the surgical arm and time is measured from time of surgery.

¥The SF-36 scores range from 0 to 100 , with higher score indicating less severe symptoms.

$\S$ The Oswestry Disability Index ranges from 1 to 100, with lower scores indicating less severe symptoms.

IThe Stenosis Bothersomeness Index and the Stenosis Frequency Index range from 0 to 24, with lower scores indicating less severe symptoms.

\|The Low Back Pain Bothersomeness Scale ranges from 0 to 6, with lower scores indicating less severe symptoms.

erative changes in isolated $\mathrm{SpS}$ patients. Previous reports may not have detected differences in DS patients as their studies were an admixture of isolated and DS patients.

In SPORT, clinical presentation of patients with varying levels of $\mathrm{SpS}$ did not differ substantially. It is known that many patients with radiographic $\mathrm{SpS}$ remain asymptomatic, ${ }^{1,2}$ so it is not surprising that multilevel $\mathrm{SpS}$ irrespective of the presence or absence of DS does not predict a worse baseline score. Mariconda et al have reported, however, a correlation with higher levels of $\mathrm{SpS}$ and higher psychosocial occupational discomfort. ${ }^{18}$ It is likely that patients present to the physicians office once a threshold of symptoms is met irrespectively of SpS degree. The correlation between severity of $\mathrm{SpS}$ and clinical symptoms awaits further study, but it is known there are many asymptomatic people with images consistent with $\mathrm{SpS}^{2}$

The advantages of the SPORT study have been described in the original SPORT lumbar SpS study. In brief, the advantages include a multicentered study, a large sample size, strict enrollment criteria, and use of multiple validated outcome scores. Although this study is a subgroup analysis of the original data, it is the first we are aware of that systematically compared independently outcomes for 1, 2, or multilevel SpS in patients with and without DS, which could influence patient outcome. Other studies included a mixed population. ${ }^{6-8,13,15,19}$

Limitations of this study include this being a secondary analysis and not the a priori hypothesis for which SPORT was designed and heterogeneity of nonsurgical treatments. The flexible nonoperative treatments allowed individualization of the treatment protocol, and more accurately reflects the treatment in most community practices.

\section{Conclusion}

In summary, this study demonstrates that patients with SpS without associated DS or scoliosis can be managed nonoperatively irrespective of the number of levels involved. If surgery is performed, the number of levels treated does not predict outcome. Overall, patients with multiple levels do as well as those with single level dis- 
ease. Patients should be reassured that despite the severity of their SpS, they should not fear that they will get worse without surgery. In contrast, patients with DS and single level SpS do better surgically than their counterparts with additional stenotic levels. Nonoperative treatment outcomes were also somewhat better in the single level patients with spondylolisthesis as compared to multilevel. This study emphasizes the importance of shared decision-making approach between the physician and patient when considering treatment for $\mathrm{SpS}$.

\section{- Key Points}

- Patients with single level stenosis with or without degenerative spondylolisthesis do not present with better clinical baseline scores than their multilevel counterparts.

- Patients with isolated single level spinal stenosis do not fare better surgically or nonoperatively than patients with multilevel stenosis treated similarly.

- Patients with single level degenerative spondylolisthesis do better surgically when the stenosis is limited to the level of slip compared to patients with additional levels of stenosis.

- This study emphasizes the importance of shared decision-making between the physician and patient when considering treatment for spinal stenosis.

\section{References}

1. Jensen MC, Brant-Zawadzki MN, Obuchowski N, et al. Magnetic resonance imaging of the lumbar spine in people without back pain. $N$ Engl J Med 1994;331:69-73.

2. Boden SD, McCowin PR, Davis DO, et al. Abnormal magnetic-resonance scans of the cervical spine in asymptomatic subjects. A prospective investigation. J Bone Joint Surg Am 1990;72:1178-84.

3. Cauchoix J, Benoist M, Chassaing V. Degenerative spondylolisthesis. Clin Orthop Relat Res 1976;115:112-29.

4. Jacobsen S, Sonne-Holm S, Rovsing H, et al. Degenerative lumbar spondylolisthesis: an epidemiological perspective: the Copenhagen Osteoarthritis Study. Spine 2007;32:120-25.

5. Rosenberg N. Degenerative spondylolisthesis: predisposing factors. J Bone Joint Surg Am 1975;57:467-74.

6. Atlas SJ, Deyo RA, Keller RB, et al. The Maine Lumbar Spine Study, Part III. 1-year outcomes of surgical and nonsurgical management of lumbar spinal stenosis. Spine 1996;21:1787-94; discussion 1794-5.

7. Atlas SJ, Keller RB, Robson D, et al. Surgical and nonsurgical management of lumbar spinal stenosis: four-year outcomes from the Maine lumbar spine study. Spine 2000;25:556-62.

8. Atlas SJ, Keller RB, Wu YA, et al. Long-term outcomes of surgical and nonsurgical management of lumbar spinal stenosis: 8 to 10 year results from the Maine lumbar spine study. Spine 2005;30:936-43.

9. Amundsen T, Weber H, Nordal H, et al. Lumbar spinal stenosis: conservative or surgical management? A prospective 10-year study. Spine 2000;25: 1424-35.

10. Weinstein JN, Tosteson TD, Lurie JD, et al. Surgical versus nonsurgical therapy for lumbar spinal stenosis. N Engl J Med 2008;358:794-810.

11. Weinstein J, Lurie JD, Tosteson TD, et al. Surgical versus nonsurgical treatment for lumbar degenerative spondylolisthesis. N Engl J Med 2007;356: 2257-70.

12. Fitzmaurice G, Laird N, Ware J. Applied Longitudinal Analysis. Philadelphia, PA: Wiley-Interscience; 2004

13. Malmivaara A, Slatis P, Heliovaara M, et al. Surgical or nonoperative treatment for lumbar spinal stenosis? A randomized controlled trial. Spine 2007; $32: 1-8$.

14. Simotas AC, Dorey FJ, Hansraj KK, et al. Nonoperative treatment for lumbar spinal stenosis. Clinical and outcome results and a 3-year survivorship analysis. Spine 2000;25:197-203; discussions 203-4.

15. Yukawa Y, Lenke LG, Tenhula J, et al. A comprehensive study of patients with surgically treated lumbar spinal stenosis with neurogenic claudication. J Bone Joint Surg Am 2002;84-A:1954-9.

16. Matsunaga S, Ijiri K, Hayashi K. Nonsurgically managed patients with degenerative spondylolisthesis: a 10 to 18 year follow-up study. J Neurosurg Spine 2000;93:194-8.

17. Vibert B, Sliva C, Herkowitz H. Treatment of instability and spondylolisthesis. Clin Orthop Relat Res 2006;443:222-7.

18. Mariconda M, Galasso O, Imbimbo L, et al. Relationship between alterations of the lumbar spine, visualized with magnetic resonance imaging, and occupational variables. Eur Spine J 2007;16:255-66.

19. Johnsson K, Rosen I, Uden A. The natural course of lumbar spinal stenosis. Clin Orthop 1992;279:82-6. 